\title{
Juvenile SLE with lupus Anticoagulant and hypoprothrominemia syndrome
}

Mohammed Olfat, $\mathrm{MD}^{1}$. Ahmed Tarawah, $\mathrm{MD}^{2}$.Doaa Mohammed ${ }^{3}$. Meyasser Olfat ${ }^{4}$

${ }^{1}$ Department of Paediatric Rheumatology, ${ }^{2}$ Department of Pediatric Hematology, ${ }^{3}$ Department of Paediatrics, ${ }^{4}$ Medical school,,${ }^{1,2,3}$ Materniy and children Hospital, MOH, ${ }^{4}$ Taibah University, Medina, Saudi Arabia

\section{Background}

- Juvenile Systemic lupus Erythematosus (JSLE) is a chronic autoimmune disease with a wide range of disease manifestations, and can lead to significant morbidity and mortality.

- Lupus anticoagulant and hypoprothrombinemia syndrome is a rare clinical entity that can occur in association with JSLE. -To our knowledge, only few cases of JSLE presenting with bleeding tendencies as a consequence of a clotting factor II (prothrombin) deficiency have been reported.

\section{Case report}

- 11 year old girl referred to our haematologist on October 2017 as a patient of suspected bleeding disorder with bilateral knee hemoarthrosis. Her history started since may 2015 when she presented to general practitioner with recurrent episodes of gingival bleeding, epistaxis, and multiple ecchymosis diagnosed as bleeding disorder and treated with fresh frozen plasma to control her bleeding. On October 2017, she had moderate gingival mucosal bleeding, moderate bilateral knee effusion and pain, generalized fatigue, eye puffiness, and significant morning stiffness. Her systemic review revealed history of intermittent claudication, nervousness, and visual hallucinations. Other systemic review were unremarkable. No family history of bleeding disorder.

- Physical examination revealed unwell, dehydrated, irritable, confused girl with puffy eyes and pallor. Her Temperature was $38.4^{\circ} \mathrm{C}$, Blood pressure 132/92, heart rate 112 beat/min, and respiratory rate of 24/min. ENT examination revealed epistaxis and gingival bleeding but no oral or nasal ulceration. Skin examination showed discoid rash, multiple ecchymosis and levido reticularis. She had polyarthritis of knees, ankles, wrists, and PIPs. Her knees showed moderate to sever effusion with tenderness, hotness and limitation of range. Her CNS examination revealed left sided hemiparesis, Other systemic examinations were unremarkable.

- Laboratory finding revealed low White blood counts (WBC) 2.8/ mm3 (4.5-10/mm3), low Haemoglobin (Hb) $6.2 \mathrm{~g} / \mathrm{dl}$ (11-14 g/dl), Platelet count 151.000/mm3(150.000-450.000), DCT positive, low Albumin 24 g/L(38-54), low Complements C3 18 mg/dl(90-180), C4 6 $\mathrm{mg} / \mathrm{dl}$ (10-40), high BUN $11.5 \mathrm{mmol} / 1$, high creatinine 125mmol/1, prolonged PT 24 (10-13), and PTT 100 (..), INR 2.18 (1-1.3), low Prothrombin II 5\% (70-120), proteinuria, microscopic hematuria, ANA, anti- dDNA, and antiphospholipid antibodies were positive, and ESR was elevated 150. Her liver function, CRP, other coagulation factors, BMA, and all infectious workup were normal. US abdomen showed bilateral nephropathy, but Echo \& chest x-ray were normal. Synovial fluid analysis from right knee showed yellowish synovial fluid and confirmed inflammatory arthritis rather than hemoarthrosis. (Figure 1)

-Finally diagnosed as JSLE with lupus anticoagulant and hypoprothrombinemia.

- Treatment: initially she received Vitamin K, Fresh Frozen Plasma, and Amicar for her uncontrolled bleeding. And as she met the diagnosis of JSLE she was started on steroids, plaquanil, low dose asprin, captopril, and cellcept. After 1 week of hospitalization she showed significant clinical and laboratory improvement.

- Discharge medications: CellCept orally $3000 \mathrm{mg}$, Prednisone $40 \mathrm{mg}$, Plaquanil 200mg, Asprin $81 \mathrm{mg}$, ranitidine 75mg, and Vitamin D3 1000 IU daily. During follow up she was doing well and went into remission on medication. On December 2017 she had herpes zoster of left upper limb and treated accordingly.

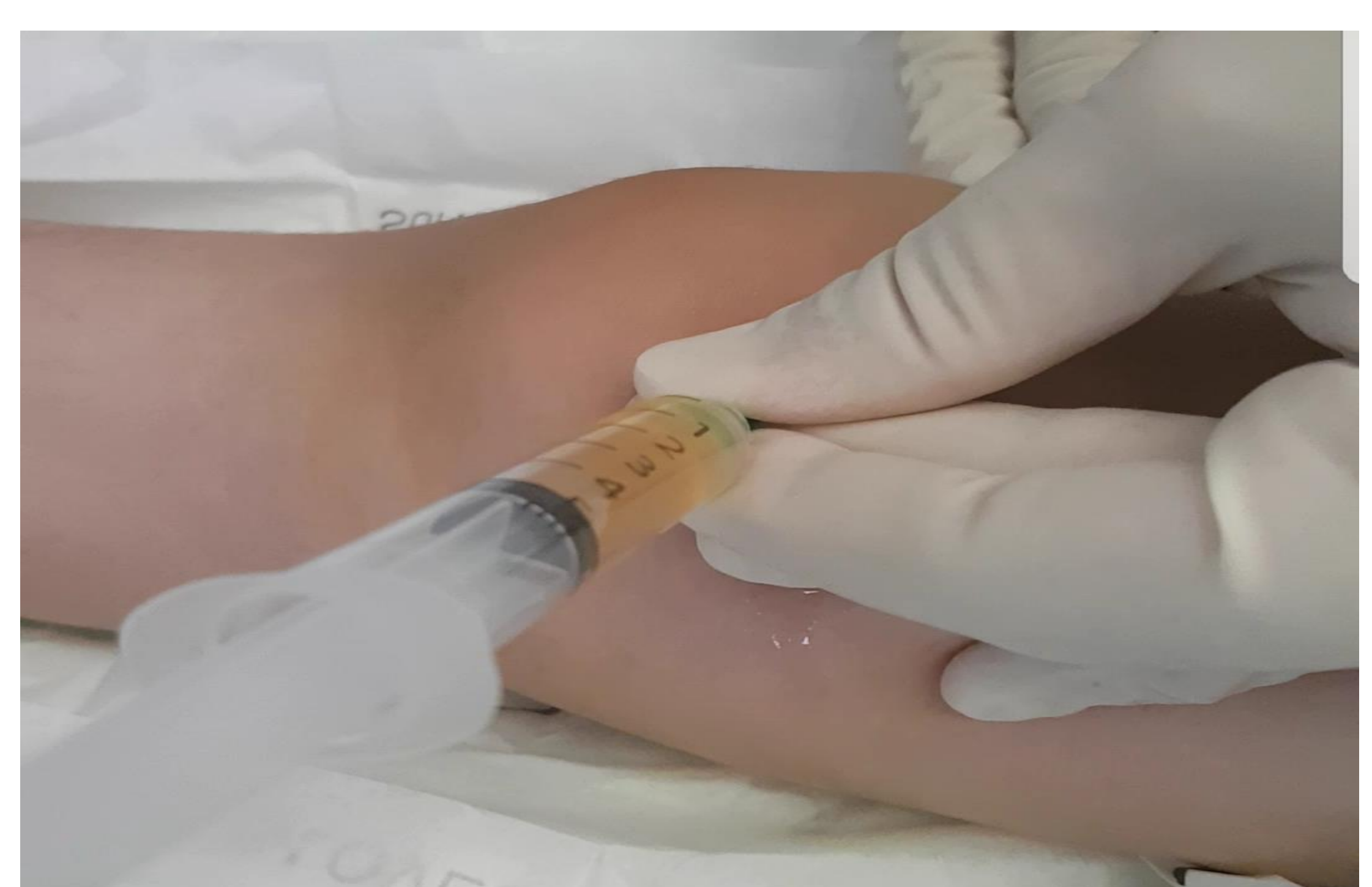

Figure 1: joint aspirate showing yellowish synovial fluid rather than blood.

\section{Discussion}

- Antigen- antibody complexes mediate much of the inflammation and tissue dysfunction associated with JSLE leading to various clinical features and complications including haematological abnormalities. Of the haematological abnormalities, patients with lupus anticoagulant commonly present with thrombosis but others may present initially with bleeding tendencies especially when they have associated prothrombin II deficiency.

- JSLE patients with lupus anticoagulant and prothrombin II deficiency have been reported to present with bleeding episodes ranging from minimal bleeding to life threatening bleeding. Our patient was similar to those cases reported in the literature. She presented initially with intermittent episodes of gingival bleeding, easy bruising, and epistaxis and later on she had other features of JSLE.

-What is more interesting in our patient is that she is found to have moderate knee effusion and thought to be hemarthrosis. However, synovial analysis confirmed inflammatory arthritis rather than hemarthrosis. In addition to that our patient had nephritis and hemiparesis. We think that her hemiparesis most likely was due to old thrombosis secondary to the presence of the antiphospholipid antibodies.

- Our patient supports the strong association between the presence of the lupus anticoagulant and prothrombin II antibodies.

- Steroids generally have been a successful first-line therapy of hypoprothrombinemia. However, Immunosuppressive medications such as azathioprine or cellcept may be needed in refractory cases. Our patient showed significant improvement after treatment with steroid in addition to vitamin $\mathrm{K}$, amicar, and fresh frozen plasma.

\section{Conclusions}

- Knowing the wide heterogeneity of disease manifestations, JSLE may present initially with bleeding tendencies due to the presence of an antibody against prothrombin II. Our case shows that when a patient present with bleeding tendency which can not be explained by the coagulation disorders particularly when he has other manifestations such as polyarthritis, nephritis, or stroke, JSLE with lupus anticoagulant and hypoprothrombinemia should be considered. After diagnosis is confirmed treatment with steroid should be initiated and when required immunosuppressive therapy may be added. 